\title{
Occurrence of dead core in catalytic particles containing immobilized enzymes: analysis for the Michaelis-Menten kinetics and assessment of numerical methods
}

\author{
Félix Monteiro Pereira ${ }^{1}$ Samuel Conceição Oliveira ${ }^{2}$
}

Received: 26 March 2016/Accepted: 23 June 2016/Published online: 30 June 2016

(C) Springer-Verlag Berlin Heidelberg 2016

\begin{abstract}
In this article, the occurrence of dead core in catalytic particles containing immobilized enzymes is analyzed for the Michaelis-Menten kinetics. An assessment of numerical methods is performed to solve the boundary value problem generated by the mathematical modeling of diffusion and reaction processes under steady state and isothermal conditions. Two classes of numerical methods were employed: shooting and collocation. The shooting method used the ode function from Scilab software. The collocation methods included: that implemented by the bvode function of Scilab, the orthogonal collocation, and the orthogonal collocation on finite elements. The methods were validated for simplified forms of the Michaelis-Menten equation (zero-order and first-order kinetics), for which analytical solutions are available. Among the methods covered in this article, the orthogonal collocation on finite elements proved to be the most robust and efficient method to solve the boundary value problem concerning Michaelis-Menten kinetics. For this enzyme kinetics, it was found that the dead core can occur when verified certain conditions of diffusion-reaction within the catalytic particle. The application of the concepts and methods presented in this study will allow for a more
\end{abstract}

Samuel Conceição Oliveira

samueloliveira@fcfar.unesp.br

Félix Monteiro Pereira

felix@dequi.eel.usp.br

1 Departamento de Engenharia Química, Escola de Engenharia de Lorena, USP-Universidade de São Paulo, Estrada Municipal do Campinho, 12602-810 Lorena, SP, Brazil

2 Departamento de Bioprocessos e Biotecnologia, Faculdade de Ciências Farmacêuticas, UNESP-Univ Estadual Paulista, Rodovia Araraquara-Jaú Km 1, 14800-903 Araraquara, SP, Brazil generalized analysis and more accurate designs of heterogeneous enzymatic reactors.

Keywords Dead core - Michaelis-Menten kinetics · Diffusion and reaction - Immobilized enzymes .

Effectiveness factor

\section{Introduction}

The main objectives of the development of new processes employing biological agents, such as cells and enzymes, include the manufacturing of new products, improvements in product quality, the minimization of environmental impacts, and cost reductions [1,2].

The bioprocesses include the transformation of raw materials (substrates) in products into bioreactors using cells or enzymes. The selection of the transformation agent and of the bioreactor operation mode depends on the assessment of the advantages and disadvantages presented by all possible configurations $[1,2]$.

The bioreactors containing immobilized enzymes are employed in several industrial processes, such as: the treatment of wastewater and the production of pharmaceuticals, chemicals, foods, beverages, biofuels, enzymes, among other bioproducts [1, 2].

The application of free enzymes within a bioreactor can cause a biocatalyst loss, which exits the reactor through the outlet flow. One of the limiting factors when using enzymes at an industrial scale is the cost of these enzymes, which can be reduced if the enzymes can be reused. The most feasible possibility of reuse is found in the immobilization of the enzymes in matrices [3].

The advantages resulting from enzyme immobilization have led to the development of new industrial processes 
employing heterogeneous enzymatic reactors. The modeling and simulation of these bioreactors is one of main steps for the design, optimization, and control of the entire process of conversion. In most cases, when modeling and simulating enzymatic reactors, it is assumed that the operating conditions are ideal, that is: plug or mixed flow pattern, constant temperature, and constant $\mathrm{pH}$. Furthermore, partitioning effects, diffusion resistance and thermal inactivation of the biocatalyst are commonly neglected in conventional modeling. However, in some cases, immobilization can cause a high diffusion resistance, requiring a detailed description of the diffusion-reaction processes within the biocatalyst.

When the diffusion resistance is quite high, the substrate concentration can be equal to zero in a given biocatalyst region, as shown in Fig. 1. In this region, known as the dead core, the reaction does not occur. Thus, the designed reactors that assume that all catalyst volume is active will present a lower performance than what is expected, justifying, in such cases, the use of an appropriate mathematical model for an accurate design of these reactors, which are subjected to mass transfer limitations.

Analysis of the dead-core occurrence for zero- and firstorder kinetics can be carried out analytically. For the

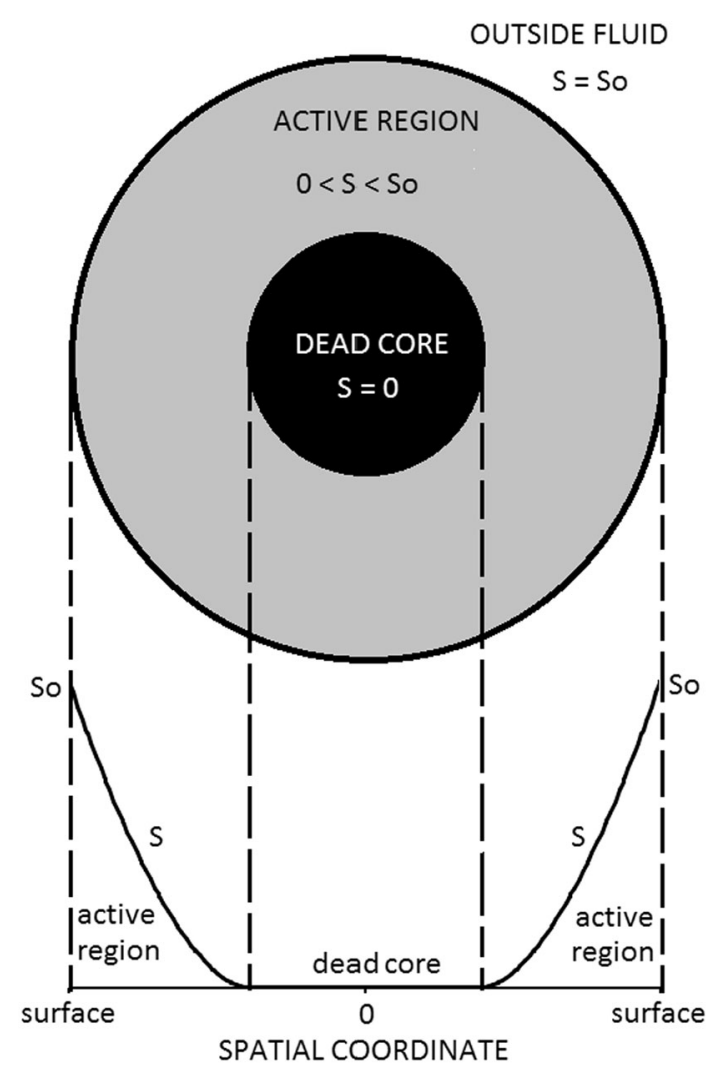

Fig. 1 Representation of dead core in a spherical particle of a biocatalyst
Michaelis-Menten kinetics, due to its non linearity, the analysis can only be conducted by applying appropriate numerical methods. The Michaelis-Menten equation successfully describes the kinetic behavior of a large number of reactions catalyzed by enzymes, thus justifying its study.

Aiming to contribute through the design and operation of bioreactors with immobilized enzymes, this study presents a methodology to analyze the dead-core occurrence for an enzymatic reaction whose kinetics is described by the Michaelis-Menten equation. For this aim, classical numerical methods were tested for the solution of boundary value problem generated by the theoretical analysis of diffusion and reaction processes.

To test the proposed numerical methodology, simplified forms of Michaelis-Menten kinetics, corresponding to zero- and first-order kinetics, were analyzed. For these cases, the analytical solutions are known for classical geometries of biocatalysts (slab, cylinder, and sphere), thus allowing one to draw comparisons with the numerical solutions. After having validated the numerical methods, the reaction-diffusion problem associated with MichaelisMenten kinetics was solved.

\section{Mathematical modeling}

The mass balance of substrate inside the particle, for steady state and isothermal conditions, is given by [4-6]:

$D_{\text {ef }}\left(\frac{\mathrm{d}^{2} S}{\mathrm{~d} X^{2}}+\frac{(\alpha-1)}{X} \frac{\mathrm{d} S}{\mathrm{~d} X}\right)=v$

In Eq. $1, S$ is the substrate concentration, $X$ is the spatial coordinate, $D_{\text {ef }}$ is the effective-diffusion coefficient of substrate inside the particle, $v$ is the rate of substrate consumption, and $\alpha$ is the geometric shape factor of the particle ( $\alpha=1$ for slab, $\alpha=2$ for cylinder and $\alpha=3$ for sphere).

Equation 1 can be given in terms of dimensionless parameters and variables, as follows:

$\frac{\mathrm{d}^{2} s}{\mathrm{~d} x^{2}}+\frac{(\alpha-1)}{x} \frac{\mathrm{d} s}{\mathrm{~d} x}=\alpha^{2} \phi^{2} \frac{v(S)}{v\left(S_{0}\right)}$

where

$x=\frac{X}{X_{L}}$

$s=\frac{S}{S_{0}}$

$\phi^{2}=\frac{X_{L}^{2}}{\alpha^{2}} \frac{v\left(S_{0}\right)}{D_{\mathrm{ef}} S_{0}}$

In Eqs. 2, 3, 4 and 5, $x$ is the dimensionless spatial coordinate, $s$ is the dimensionless substrate concentration, $X_{L}$ is the half value of thickness for slab and the radius for 
cylinder and sphere, $S_{O}$ is the substrate concentration on the particle surface, and $\phi$ is the Thiele modulus.

Equation (2) is subjected to the following boundary conditions:

$$
\begin{aligned}
\frac{\mathrm{d} s}{\mathrm{~d} x} & =0 \text { at } x \\
& =a, \text { for } \phi>\phi_{\text {crit }}(\text { problem with dead-core occurrence })
\end{aligned}
$$

$$
\begin{aligned}
\frac{\mathrm{d} s}{\mathrm{~d} x} & =0 \quad \text { at } x \\
& =0, \text { for } \phi \leq \phi_{\text {crit }}(\text { problem without dead-core occurrence) }
\end{aligned}
$$

$s=1 \quad$ at $\quad x=1$

In Eq. 6, $a$ is the dead-core position (dimensionless), and $\phi_{\text {crit }}$ is the critical value of the Thiele modulus above which the dead core occurs.

Equation 9 can be used to calculate the internal effectiveness factor $(\eta)[6,7]$, a parameter ranging from 0 to 1 that indicates how much diffusion resistance controls the rate of reaction.

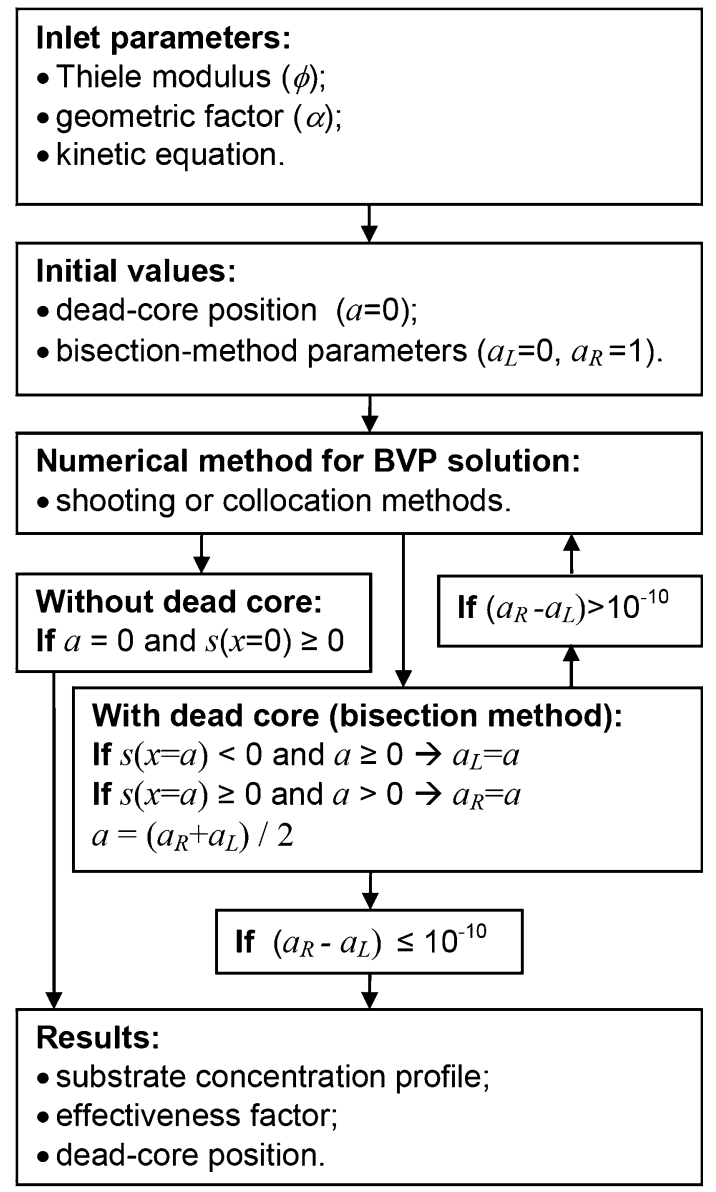

Fig. 2 Flowchart to calculate the concentration profiles, effectiveness factor, and dead-core position $\eta=\left.\frac{1}{\alpha \phi^{2}} \frac{\mathrm{d} s}{\mathrm{~d} x}\right|_{x=1}$

Equation (10) shows the substrate consumption rate given by Michaelis-Menten kinetics [4, 5]:

$v(S)=\frac{v_{\max } S}{K+S}$

where, $K$ is the Michaellis-Menten constant, and $v_{\max }$ is the maximum rate of substrate consumption.

The Michaelis-Menten equation has the following asymptotic properties [4, 5]:

(a) For low substrate concentrations $(s \ll K)$, it can be approximated to first-order kinetics:

$v(S)=k_{1} S$, where $k_{1}=v_{\max } / K$

(b) For high substrate concentrations $(s \gg K)$, it can be approximated to zero-order kinetics:

$v(S)=k_{0}$, where $k_{0}=v_{\max }$

Thus, introducing Eqs. 10, 11 and 12 into Eq. (2):

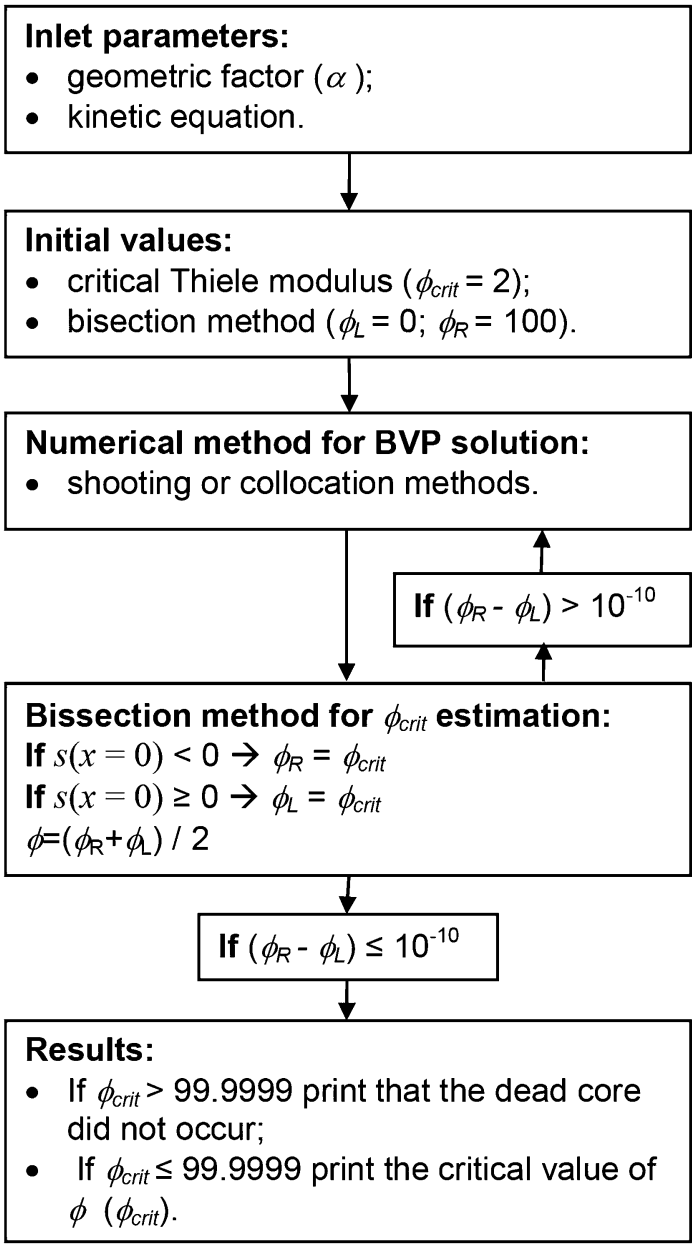

Fig. 3 Flowchart for the numerical determination of the critical Thiele modulus $\left(\phi_{\text {crit }}\right)$ 
$\frac{\mathrm{d}^{2} s}{\mathrm{~d} x^{2}}+\frac{(\alpha-1)}{x} \frac{\mathrm{d} s}{\mathrm{~d} x}=\alpha^{2} \phi^{2} \frac{(1+\beta) s}{\beta+s}$

$\frac{\mathrm{d}^{2} s}{\mathrm{~d} x^{2}}+\frac{(\alpha-1)}{x} \frac{\mathrm{d} s}{\mathrm{~d} x}=\alpha^{2} \phi^{2} s$

$\frac{\mathrm{d}^{2} s}{\mathrm{~d} x^{2}}+\frac{(\alpha-1)}{x} \frac{\mathrm{d} s}{\mathrm{~d} x}=\alpha^{2} \phi^{2}$

In Eq. $13, \beta$ is the dimensionless Michaelis-Menten constant $\left(\beta=K / S_{0}\right)$.

The exact analytical solutions of Eqs. 14 and 15 can be found using the software Mathematica [8]. These solutions were used to assess the accuracy of the numerical methods applied in this study to solve Eq. 13.
The analytical solutions for the zero-order kinetics (Eq. 15), subjected to the boundary conditions given by Eqs. 6, 7 and 8, for slab (Eqs. 16 and 17), cylinder (Eqs. 18 and 19), and sphere (Eqs. 20 and 21) particles, are presented as follows.

$s=\frac{1}{2}\left\{2+\left[x^{2}-1-2 a(x-1)\right] \phi^{2}\right\}$, for $\phi>\phi_{\text {crit }}$

$s=\frac{1}{2}\left[2+\left(x^{2}-1\right) \phi^{2}\right]$, for $\phi \leq \phi_{\text {crit }}$

$s=1+\left(x^{2}-1\right) \phi^{2}-2 a^{2} \phi^{2} \ln (x)$, for $\phi>\phi_{\text {crit }}$

$s=1+\left(x^{2}-1\right) \phi^{2}$, for $\phi \leq \phi_{\text {crit }}$
Table 1 Results from the shooting method for the BVP solution for zero- and first-order kinetics

\begin{tabular}{|c|c|c|c|c|c|c|c|c|c|c|}
\hline Order & $\alpha$ & $\phi$ & $\phi_{a}$ & $\phi_{n}$ & error $_{\phi}$ & $a$ & $\eta_{a}$ & $\eta_{n}$ & error $_{\eta}$ & error $_{s}$ \\
\hline 0 & 1 & 0.5 & $\sqrt{2}$ & 1.4142 & $1.4 \times 10^{-13}$ & 0 & 1 & 1 & 0 & $2.5 \times 10^{-14}$ \\
\hline 0 & 1 & 1 & $\sqrt{2}$ & 1.4142 & $1.4 \times 10^{-13}$ & 0 & 1 & 1 & 0 & $9.9 \times 10^{-14}$ \\
\hline 0 & 1 & 2 & $\sqrt{2}$ & 1.4142 & $1.4 \times 10^{-13}$ & 0.2929 & 0.7071 & 0.7071 & $8.8 \times 10^{-13}$ & $2.5 \times 10^{-12}$ \\
\hline 0 & 1 & 4 & $\sqrt{2}$ & 1.4142 & $1.4 \times 10^{-13}$ & 0.6464 & 0.3536 & 0.3536 & $1.1 \times 10^{-13}$ & $6.2 \times 10^{-13}$ \\
\hline 0 & 1 & 8 & $\sqrt{2}$ & 1.4142 & $1.4 \times 10^{-13}$ & 0.8232 & 0.1768 & 0.1768 & $1.4 \times 10^{-14}$ & $1.6 \times 10^{-13}$ \\
\hline 0 & 2 & 0,5 & 1 & 1.0000 & 0 & 0 & 1 & 1 & 0 & $5.0 \times 10^{-14}$ \\
\hline 0 & 2 & 1 & 1 & 1.0000 & 0 & 0 & 1 & 1 & $1.1 \times 10^{-10}$ & $9.0 \times 10^{-11}$ \\
\hline 0 & 2 & 2 & 1 & 1.0000 & 0 & 0.6184 & 0.6176 & 0.6176 & $4.8 \times 10^{-9}$ & $4.3 \times 10^{-9}$ \\
\hline 0 & 2 & 4 & 1 & 1.0000 & 0 & 0.8173 & 0.3320 & 0.3320 & $6.3 \times 10^{-10}$ & $1.0 \times 10^{-8}$ \\
\hline 0 & 2 & 8 & 1 & 1.0000 & 0 & 0.9102 & 0.1715 & 0.1715 & $6.4 \times 10^{-10}$ & $8.3 \times 10^{-9}$ \\
\hline 0 & 3 & 0.5 & $\sqrt{6} / 3$ & 0.8165 & $8.1 \times 10^{-14}$ & 0 & 1 & 1 & $3.7 \times 10^{-14}$ & $7.7 \times 10^{-14}$ \\
\hline 0 & 3 & 1 & $\sqrt{6} / 3$ & 0.8165 & $8.1 \times 10^{-14}$ & 0.3870 & 0.9421 & 0.9421 & $5.0 \times 10^{-8}$ & $1.1 \times 10^{-8}$ \\
\hline 0 & 3 & 2 & $\sqrt{6} / 3$ & 0.8165 & $8.1 \times 10^{-14}$ & 0.7409 & 0.5934 & 0.5934 & $1.4 \times 10^{-8}$ & $8.3 \times 10^{-9}$ \\
\hline 0 & 3 & 4 & $\sqrt{6} / 3$ & 0.8165 & $8.1 \times 10^{-14}$ & 0.8770 & 0.3255 & 0.3255 & $4.8 \times 10^{-10}$ & $8.9 \times 10^{-9}$ \\
\hline 0 & 3 & 8 & $\sqrt{6} / 3$ & 0.8165 & $8.1 \times 10^{-14}$ & 0.9399 & 0.1698 & 0.1698 & $2.7 \times 10^{-10}$ & $5.4 \times 10^{-9}$ \\
\hline 1 & 1 & 0.5 & a & $\mathrm{b}$ & a & 0 & 0.9242 & 0.9242 & $8.4 \times 10^{-8}$ & $9.5 \times 10^{-8}$ \\
\hline 1 & 1 & 1 & $\mathrm{a}$ & b & a & 0 & 0.7616 & 0.7616 & $5.0 \times 10^{-9}$ & $7.1 \times 10^{-8}$ \\
\hline 1 & 1 & 2 & a & $\mathrm{b}$ & a & 0 & 0.4820 & 0.4820 & $5.9 \times 10^{-9}$ & $5.3 \times 10^{-8}$ \\
\hline 1 & 1 & 4 & a & b & a & 0 & 0.2498 & 0.2498 & $4.9 \times 10^{-11}$ & $1.5 \times 10^{-8}$ \\
\hline 1 & 1 & 8 & a & b & a & 0 & 0.1250 & 0.1250 & $2.8 \times 10^{-14}$ & $2.7 \times 10^{-8}$ \\
\hline 1 & 2 & 0.5 & $\mathrm{a}$ & $\mathrm{b}$ & a & 0 & 0.8928 & 0.8928 & $5.9 \times 10^{-9}$ & $3.6 \times 10^{-8}$ \\
\hline 1 & 2 & 1 & a & $\mathrm{b}$ & a & 0 & 0.6978 & 0.6978 & $1.3 \times 10^{-8}$ & $1.1 \times 10^{-7}$ \\
\hline 1 & 2 & 2 & $\mathrm{a}$ & b & a & 0 & 0.4318 & 0.4318 & $9.0 \times 10^{-10}$ & $2.2 \times 10^{-8}$ \\
\hline 1 & 2 & 4 & $\mathrm{a}$ & $\mathrm{b}$ & a & 0 & 0.2338 & 0.2338 & $2.8 \times 10^{-10}$ & $3.1 \times 10^{-8}$ \\
\hline 1 & 2 & 8 & $\mathrm{a}$ & $\mathrm{b}$ & a & 0 & 0.1210 & 0.1210 & $3.6 \times 10^{-11}$ & $1.8 \times 10^{-8}$ \\
\hline 1 & 3 & 0.5 & a & $\mathrm{b}$ & a & 0 & 0.8762 & 0.8762 & $1.4 \times 10^{-8}$ & $4.1 \times 10^{-8}$ \\
\hline 1 & 3 & 1 & a & $\mathrm{b}$ & a & 0 & 0.6716 & 0.6716 & $2.6 \times 10^{-8}$ & $1.8 \times 10^{-7}$ \\
\hline 1 & 3 & 2 & a & $\mathrm{b}$ & a & 0 & 0.4167 & 0.4167 & $9.8 \times 10^{-9}$ & $2.1 \times 10^{-7}$ \\
\hline 1 & 3 & 4 & a & $\mathrm{b}$ & a & 0 & 0.2292 & 0.2292 & $1.4 \times 10^{-9}$ & $1.6 \times 10^{-7}$ \\
\hline 1 & 3 & 8 & $\mathrm{a}$ & $\mathrm{b}$ & $\mathrm{a}$ & 0 & 0.1198 & 0.1198 & $4.3 \times 10^{-11}$ & $3.0 \times 10^{-8}$ \\
\hline
\end{tabular}

${ }^{a}$ Dead-core does not occur for the analytical solution of first-order kinetics [7]

b Method is divergent for high values of Thiele's modulus (determination of $\phi_{n}$ is not possible) 
$s=1-\frac{3\left[2 a^{3}(x-1)+x-x^{3}\right] \phi^{2}}{2 x}$, for $\phi>\phi_{\text {crit }}$

$s=1-\frac{3}{2}\left(1-x^{2}\right) \phi^{2}$, for $\phi \leq \phi_{\text {crit }}$

Since the dead core occurs for $\phi>\phi_{\text {crit }}$, the values of $\phi_{\text {crit }}$ for the zero-order kinetics determined analytically are as follows: $\phi_{\text {crit }}=\sqrt{2}$ (slab), $\phi_{\text {crit }}=1$ (cylinder), and $\phi_{\text {crit }}=\sqrt{6} / 3$ (sphere). The value of $a$ is determined by taking $s=0$ and $x=a$ in Eqs. 16, 18 and 20 .

For zero-order kinetics, the effectiveness factor can be calculated analytically by Eq. 22 for the three classical geometries.

$\eta=1-a^{\alpha}$
The analytical solutions for first-order kinetics (Eq. 14), subjected to the boundary conditions given by Eqs. 7 and 8, are presented as follows for slab (Eq. 23), cylinder (Eq. 24), and sphere (Eq. 25) particles [8-10].

$s=\frac{\cosh (x \phi)}{\cosh (\phi)}$

$s=\frac{\mathrm{I}_{0}(2 x \phi)}{\mathrm{I}_{0}(2 \phi)}$

$s=\frac{\sinh (3 x \phi)}{x \sinh (3 \phi)}$

For first-order kinetics, the effectiveness factor can be calculated analytically as follows for slab (Eq. 26), cylinder (Eq. 27), and sphere (Eq. 28) [8-10].
Table 2 Results from the orthogonal collocation method for the BVP solution for zeroorder and first-order kinetics

\begin{tabular}{|c|c|c|c|c|c|c|c|c|c|c|}
\hline Order & $\alpha$ & $\phi$ & $\phi_{a}$ & $\phi_{n}$ & error $_{\phi}$ & $a$ & $\eta_{a}$ & $\eta_{n}$ & error $_{\eta}$ & error $_{s}$ \\
\hline 0 & 1 & 0.5 & $\sqrt{2}$ & 1.4142 & $2.9 \times 10^{-8}$ & 0 & 1 & 1 & $2.5 \times 10^{-6}$ & $6.0 \times 10^{-7}$ \\
\hline 0 & 1 & 1 & $\sqrt{2}$ & 1.4142 & $2.9 \times 10^{-8}$ & 0 & 1 & 1 & $5.0 \times 10^{-7}$ & $4.0 \times 10^{-7}$ \\
\hline 0 & 1 & 2 & $\sqrt{2}$ & 1.4142 & $2.9 \times 10^{-8}$ & 0.2929 & 0.7071 & 0.7071 & $2.0 \times 10^{-7}$ & $3.5 \times 10^{-8}$ \\
\hline 0 & 1 & 4 & $\sqrt{2}$ & 1.4142 & $2.9 \times 10^{-8}$ & 0.6464 & 0.3536 & 0.3536 & $8.4 \times 10^{-8}$ & $3.5 \times 10^{-8}$ \\
\hline 0 & 1 & 8 & $\sqrt{2}$ & 1.4142 & $2.9 \times 10^{-8}$ & 0.8232 & 0.1768 & 0.1768 & $4.2 \times 10^{-8}$ & $3.5 \times 10^{-8}$ \\
\hline 0 & 2 & 0.5 & 1 & 1.0000 & $1.0 \times 10^{-7}$ & 0 & 1 & 1 & $8.4 \times 10^{-6}$ & $3.0 \times 10^{-7}$ \\
\hline 0 & 2 & 1 & 1 & 1.0000 & $1.0 \times 10^{-7}$ & 0 & 1 & 1 & $2.0 \times 10^{-6}$ & $2.0 \times 10^{-7}$ \\
\hline 0 & 2 & 2 & 1 & 1.0000 & $1.0 \times 10^{-7}$ & 0.6184 & 0.6176 & 0.6176 & $2.2 \times 10^{-5}$ & $7.5 \times 10^{-5}$ \\
\hline 0 & 2 & 4 & 1 & 1.0000 & $1.0 \times 10^{-7}$ & 0.8173 & 0.3320 & 0.3320 & $3.9 \times 10^{-6}$ & $2.6 \times 10^{-5}$ \\
\hline 0 & 2 & 8 & 1 & 1.0000 & $1.0 \times 10^{-7}$ & 0.9102 & 0.1715 & 0.1715 & $7.0 \times 10^{-7}$ & $1.1 \times 10^{-5}$ \\
\hline 0 & 3 & 0.5 & $\sqrt{6} / 3$ & 0.8165 & $4.0 \times 10^{-8}$ & 0 & 1 & 1 & $4.4 \times 10^{-6}$ & $2.0 \times 10^{-7}$ \\
\hline 0 & 3 & 1 & $\sqrt{6} / 3$ & 0.8165 & $4.0 \times 10^{-8}$ & 0.3814 & 0.9421 & 0.9418 & $2.2 \times 10^{-4}$ & $8.1 \times 10^{-4}$ \\
\hline 0 & 3 & 2 & $\sqrt{6} / 3$ & 0.8165 & $4.0 \times 10^{-8}$ & 0.7402 & 0.5934 & 0.5933 & $4.5 \times 10^{-5}$ & $1.7 \times 10^{-4}$ \\
\hline 0 & 3 & 4 & $\sqrt{6} / 3$ & 0.8165 & $4.0 \times 10^{-8}$ & 0.8769 & 0.3255 & 0.3255 & $1.0 \times 10^{-5}$ & $6.4 \times 10^{-5}$ \\
\hline 0 & 3 & 8 & $\sqrt{6} / 3$ & 0.8165 & $4.0 \times 10^{-8}$ & 0.9398 & 0.1698 & 0.1698 & $2.7 \times 10^{-6}$ & $2.8 \times 10^{-5}$ \\
\hline 1 & 1 & 0.5 & a & $\mathrm{b}$ & $\mathrm{a}$ & 0 & 0.9242 & 0.9242 & $2.8 \times 10^{-6}$ & $5.0 \times 10^{-7}$ \\
\hline 1 & 1 & 1 & $\mathrm{a}$ & $\mathrm{b}$ & $\mathrm{a}$ & 0 & 0.7616 & 0.7616 & $7.0 \times 10^{-7}$ & $4.0 \times 10^{-7}$ \\
\hline 1 & 1 & 2 & $\mathrm{a}$ & $\mathrm{b}$ & $\mathrm{a}$ & 0 & 0.4820 & 0.4820 & $1.0 \times 10^{-7}$ & $5.0 \times 10^{-6}$ \\
\hline 1 & 1 & 4 & $\mathrm{a}$ & $\mathrm{b}$ & $\mathrm{a}$ & 0 & 0.2498 & 0.2498 & $5.4 \times 10^{-9}$ & $2.5 \times 10^{-5}$ \\
\hline 1 & 1 & 8 & $\mathrm{a}$ & $\mathrm{b}$ & $\mathrm{a}$ & 0 & 0.1250 & 0.1250 & $1.5 \times 10^{-8}$ & $1.3 \times 10^{-4}$ \\
\hline 1 & 2 & 0.5 & $\mathrm{a}$ & $\mathrm{b}$ & $\mathrm{a}$ & 0 & 0.8928 & 0.8928 & $8.2 \times 10^{-6}$ & $5.0 \times 10^{-7}$ \\
\hline 1 & 2 & 1 & a & $\mathrm{b}$ & $\mathrm{a}$ & 0 & 0.6978 & 0.6978 & $2.0 \times 10^{-6}$ & $2.8 \times 10^{-6}$ \\
\hline 1 & 2 & 2 & $\mathrm{a}$ & $\mathrm{b}$ & $\mathrm{a}$ & 0 & 0.4318 & 0.4318 & $5.0 \times 10^{-7}$ & $1.7 \times 10^{-5}$ \\
\hline 1 & 2 & 4 & a & $\mathrm{b}$ & $\mathrm{a}$ & 0 & 0.2338 & 0.2338 & $1.0 \times 10^{-7}$ & $1.0 \times 10^{-4}$ \\
\hline 1 & 2 & 8 & a & $\mathrm{b}$ & $\mathrm{a}$ & 0 & 0.1210 & 0.1210 & $4.0 \times 10^{-7}$ & $4.8 \times 10^{-4}$ \\
\hline 1 & 3 & 0.5 & $\mathrm{a}$ & $\mathrm{b}$ & $\mathrm{a}$ & 0 & 0.8762 & 0.8762 & $4.2 \times 10^{-6}$ & $6.0 \times 10^{-7}$ \\
\hline 1 & 3 & 1 & $\mathrm{a}$ & $\mathrm{b}$ & a & 0 & 0.6716 & 0.6716 & $1.2 \times 10^{-6}$ & $5.2 \times 10^{-6}$ \\
\hline 1 & 3 & 2 & $\mathrm{a}$ & $\mathrm{b}$ & $\mathrm{a}$ & 0 & 0.4167 & 0.4167 & $4.0 \times 10^{-7}$ & $3.8 \times 10^{-5}$ \\
\hline 1 & 3 & 4 & a & $\mathrm{b}$ & $\mathrm{a}$ & 0 & 0.2292 & 0.2292 & $2.0 \times 10^{-7}$ & $2.1 \times 10^{-4}$ \\
\hline 1 & 3 & 8 & a & $\mathrm{b}$ & $\mathrm{a}$ & 0 & 0.1198 & 0.1198 & $1.4 \times 10^{-5}$ & $9.9 \times 10^{-4}$ \\
\hline
\end{tabular}

${ }^{a}$ Dead-core does not occur for the analytical solution of first-order kinetics [7]

b Converges to the maximum value of $\phi_{n}\left(\phi_{n}=100\right)$ for the bisection method 
$\eta=\frac{\tanh (\phi)}{\phi}$

$\eta=\frac{I_{1}(2 \phi)}{\phi I_{0}(2 \phi)}$

$\eta=\frac{1}{\phi \tanh (3 \phi)}-\frac{1}{3 \phi^{2}}$

In Eqs. 24 and 27, $I_{0}$ and $I_{1}$ are the modified Bessel functions of first kind and zero $\left(I_{0}\right)$ and one $\left(I_{1}\right)$ orders.

For the dead-core occurrence problem, incorporating the Michaelis-Menten kinetics, numerical and exact analytical solutions are not reported in the literature. For problems without dead-core occurrence, some approximated analytical solutions are proposed [11-14]. As the dead core can occur for high values of the Thiele modulus, a reliable numerical method must be used for both the calculus of internal effectiveness and the search/validation of approximated analytical solutions for the problems including dead-core occurrence.

In this article, different numerical methods were applied to calculate the substrate concentration profile and internal effectiveness factor for Michaelis-Menten kinetics, taking the dead-core occurrence into account.

\section{Numerical methodology}

A computer program was developed in the Scilab software to solve the boundary value problem (BVP) given by
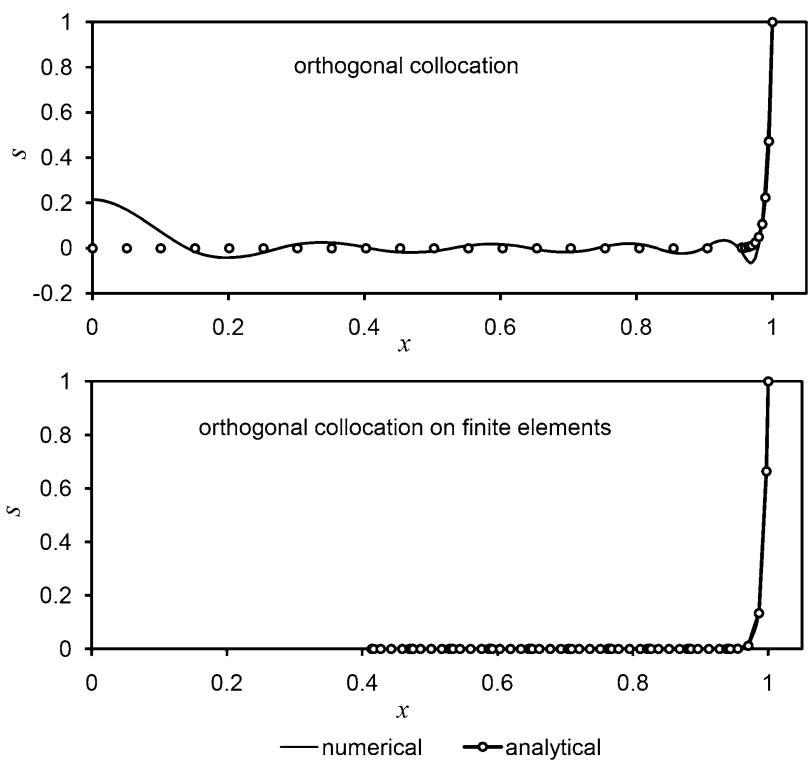

Fig. 4 Profile of substrate concentration $(\phi=50, \alpha=3$, first-order kinetics) calculated by orthogonal collocation method and orthogonal collocation method on finite elements
Eqs. 2, 6, 7 and 8, and several numerical methods, including shooting and collocation methods, were tested.

The shooting methods used the fsolve function of Scilab, which is based on Newton's method, coupled with the ode function, which is the standard function to integrate explicit ordinary differential equation systems. The ode function is an interface to several solvers. Hence, the present study tested the following methods:

- Nonstiff predictor-corrector Adams [15-17];

- Stiff backward differentiation formula [15-17];

- Adaptive Runge-Kutta of 4th order [18-20];

- Shampine and Watts program based on Fehlberg's Runge-Kutta pair of orders 4 and 5 [18-20].

Among the collocation methods used in this study, that implemented by the bvode function of Scilab solves a multi-point boundary value problem for a mixed order system of ordinary differential equations, using algorithms from the literature [21-24]. Other collocation methods used in this study included the methods of orthogonal collocation and orthogonal collocation on finite elements [25, 26], which were implemented in Scilab using the following functions:

- legendre: to obtain the Legendre polynomials;

- lsqrsolve, poly, and roots: to calculate the collocation points;

- fsolve (Newton's method): to calculate the substrate concentration on collocation points.

Figure 2 shows the flowchart to calculate the concentration profile, effectiveness factor, and dead-core position.

Figure 3 presents the flowchart for the numerical determination of the critical Thiele modulus.

The BVP solution method coupled with the bisection method was applied to determine the dead-core position (a) and the critical Thiele modulus ( $\left.\phi_{\text {crit }}\right)$.

In Figs. 2 and 3, $a_{L}, a_{R}, \phi_{L}$, and $\phi_{R}$ are the minimum or left (L-subscript) and maximum or right (R-subscript) boundary values used in the bisection method to determine $a$ and $\phi_{\text {crit }}$ parameters.

Based on the flowcharts presented in Figs. 2 and 3, three numerical methods were applied to the solution of the BVP: shooting, orthogonal collocation, and orthogonal collocation on finite elements.

\section{Results and discussion}

From the results obtained using the developed computer programs, the numerical and analytical solutions for the simplified forms of Michaelis-Menten equation were compared to assess the validity and accuracy of the 
Table 3 Results from orthogonal collocation method on finite elements for the BVP solution for zero- and first-order kinetics

\begin{tabular}{|c|c|c|c|c|c|c|c|c|c|c|}
\hline Order & $\alpha$ & $\phi$ & $\phi_{a}$ & $\phi_{n}$ & error $_{\phi}$ & $a$ & $\eta_{a}$ & $\eta_{n}$ & error $_{\eta}$ & error $_{s}$ \\
\hline 0 & 1 & 0.5 & $\sqrt{2}$ & 1.4142 & $2.2 \times 10^{-11}$ & 0 & 1 & 1 & $5.7 \times 10^{-11}$ & $9.9 \times 10^{-12}$ \\
\hline 0 & 1 & 1 & $\sqrt{2}$ & 1.4142 & $2.2 \times 10^{-11}$ & 0 & 1 & 1 & $1.1 \times 10^{-11}$ & $6.9 \times 10^{-12}$ \\
\hline 0 & 1 & 2 & $\sqrt{2}$ & 1.4142 & $2.2 \times 10^{-11}$ & 0.2929 & 0.7071 & 0.7071 & $2.6 \times 10^{-11}$ & $2.7 \times 10^{-12}$ \\
\hline 0 & 1 & 4 & $\sqrt{2}$ & 1.4142 & $2.2 \times 10^{-11}$ & 0.6464 & 0.3536 & 0.3536 & $2.7 \times 10^{-11}$ & $2.6 \times 10^{-12}$ \\
\hline 0 & 1 & 8 & $\sqrt{2}$ & 1.4142 & $2.2 \times 10^{-11}$ & 0.8232 & 0.1768 & 0.1768 & $2.8 \times 10^{-11}$ & $2.8 \times 10^{-12}$ \\
\hline 0 & 2 & 0.5 & 1 & 1.0000 & $1.3 \times 10^{-11}$ & 0 & 1 & 1 & $9.0 \times 10^{-12}$ & $4.0 \times 10^{-12}$ \\
\hline 0 & 2 & 1 & 1 & 1.0000 & $1.3 \times 10^{-11}$ & 0 & 1 & 1 & $2.0 \times 10^{-12}$ & $2.0 \times 10^{-12}$ \\
\hline 0 & 2 & 2 & 1 & 1.0000 & $1.3 \times 10^{-11}$ & 0.6184 & 0.6176 & 0.6176 & $3.6 \times 10^{-11}$ & $2.8 \times 10^{-12}$ \\
\hline 0 & 2 & 4 & 1 & 1.0000 & $1.3 \times 10^{-11}$ & 0.8173 & 0.3320 & 0.3320 & $3.3 \times 10^{-11}$ & $2.6 \times 10^{-12}$ \\
\hline 0 & 2 & 8 & 1 & 1.0000 & $1.3 \times 10^{-11}$ & 0.9102 & 0.1715 & 0.1715 & $3.8 \times 10^{-11}$ & $2.7 \times 10^{-12}$ \\
\hline 0 & 3 & 0.5 & $\sqrt{6} / 3$ & 0.8165 & $2.7 \times 10^{-12}$ & 0 & 1 & 1 & $2.9 \times 10^{-12}$ & $2.6 \times 10^{-12}$ \\
\hline 0 & 3 & 1 & $\sqrt{6} / 3$ & 0.8165 & $2.7 \times 10^{-12}$ & 0.3869 & 0.9421 & 0.9421 & $8.8 \times 10^{-14}$ & $7.1 \times 10^{-11}$ \\
\hline 0 & 3 & 2 & $\sqrt{6} / 3$ & 0.8165 & $2.7 \times 10^{-12}$ & 0.7402 & 0.5934 & 0.5934 & $1.1 \times 10^{-12}$ & $2.6 \times 10^{-12}$ \\
\hline 0 & 3 & 4 & $\sqrt{6} / 3$ & 0.8165 & $2.7 \times 10^{-12}$ & 0.8769 & 0.3255 & 0.3255 & $7.7 \times 10^{-12}$ & $2.6 \times 10^{-12}$ \\
\hline 0 & 3 & 8 & $\sqrt{6} / 3$ & 0.8165 & $2.7 \times 10^{-12}$ & 0.9398 & 0.1698 & 0.1698 & $3.0 \times 10^{-11}$ & $2.6 \times 10^{-12}$ \\
\hline 1 & 1 & 0.5 & a & 86.9590 & $\mathrm{a}$ & 0 & 0.9242 & 0.9242 & $5.1 \times 10^{-11}$ & $9.1 \times 10^{-12}$ \\
\hline 1 & 1 & 1 & a & 86.9590 & a & 0 & 0.7616 & 0.7616 & $8.4 \times 10^{-12}$ & $5.7 \times 10^{-12}$ \\
\hline 1 & 1 & 2 & a & 86.9590 & $\mathrm{a}$ & 0 & 0.4820 & 0.4820 & $8.5 \times 10^{-13}$ & $1.7 \times 10^{-12}$ \\
\hline 1 & 1 & 4 & a & 86.9590 & a & 0 & 0.2498 & 0.2498 & $7.6 \times 10^{-14}$ & $3.0 \times 10^{-11}$ \\
\hline 1 & 1 & 8 & a & 86.9590 & $\mathrm{a}$ & 0 & 0.1250 & 0.1250 & $1.1 \times 10^{-12}$ & $3.3 \times 10^{-9}$ \\
\hline 1 & 2 & 0.5 & a & 43.9220 & a & 0 & 0.8928 & 0.8928 & $7.0 \times 10^{-12}$ & $3.4 \times 10^{-12}$ \\
\hline 1 & 2 & 1 & a & 43.9220 & $\mathrm{a}$ & 0 & 0.6978 & 0.6978 & $1.1 \times 10^{-12}$ & $1.7 \times 10^{-12}$ \\
\hline 1 & 2 & 2 & a & 43.9220 & a & 0 & 0.4318 & 0.4318 & $1.2 \times 10^{-13}$ & $1.8 \times 10^{-11}$ \\
\hline 1 & 2 & 4 & a & 43.9220 & a & 0 & 0.2338 & 0.2338 & $2.1 \times 10^{-12}$ & $2.4 \times 10^{-9}$ \\
\hline 1 & 2 & 8 & a & 43.9220 & a & 0 & 0.1210 & 0.1210 & $8.5 \times 10^{-10}$ & $2.6 \times 10^{-7}$ \\
\hline 1 & 3 & 0.5 & a & 29.5413 & a & 0 & 0.8762 & 0.8762 & $2.0 \times 10^{-12}$ & $2.2 \times 10^{-12}$ \\
\hline 1 & 3 & 1 & a & 29.5413 & a & 0 & 0.6716 & 0.6716 & $2.3 \times 10^{-13}$ & $1.4 \times 10^{-12}$ \\
\hline 1 & 3 & 2 & a & 29.5413 & a & 0 & 0.4167 & 0.4167 & $2.4 \times 10^{-13}$ & $2.2 \times 10^{-10}$ \\
\hline 1 & 3 & 4 & a & 29.5413 & a & 0 & 0.2292 & 0.2292 & $1.0 \times 10^{-10}$ & $3.1 \times 10^{-8}$ \\
\hline 1 & 3 & 8 & $\mathrm{a}$ & 29.5413 & a & 0 & 0.1198 & 0.1198 & $3.3 \times 10^{-8}$ & $3.1 \times 10^{-6}$ \\
\hline
\end{tabular}

${ }^{a}$ Dead-core does not occur for the analytical solution of the first-order kinetics [7] proposed numerical methodologies. For this comparison, the following parameters were analyzed:

- Internal effectiveness factor calculated by numerical and analytical solutions and the absolute difference between them: $\eta_{n}, \eta_{a}$, error $_{\eta}=\left|\eta_{a}-\eta_{n}\right|$;

- Critical Thiele modulus calculated by numerical and analytical solutions and the absolute difference between them: $\phi_{n}, \phi_{a}, \operatorname{error}_{\phi}=\left|\phi_{a}-\phi_{n}\right|$

- Maximum absolute difference between the values of dimensionless substrate concentration calculated by numerical and analytical solutions: error $_{s}$

The results for each applied method are presented separately as follows.

\section{Shooting methods}

Table 1 shows the results obtained from the shooting methods. The results were the same for all available methods in the ode function of Scilab.

Results on Table 1 indicate that the shooting methods coupled with the ode function of Scilab were able to solve the BVP for a wide range of Thiele modulus. However, for high values of Thiele modulus, all methods available in the ode function proved to be divergent when the first-order kinetics was analyzed. This result suggests that another methodology must be employed to solve the nonlinear boundary value problems. 


\section{Collocation methods}

Similar results (Table 2) were obtained using two collocation methods: the orthogonal collocation and that implemented by the bvode function of Scilab.

Table 2 shows a low accuracy to define the internal effectiveness factor at high values of the Thiele modulus. For high values from the Thiele modulus, the calculated values of effectiveness factor do not agree with the analytical values. This fact was associated with the inconsistent concentration profiles obtained for these cases, as clearly shown in Fig. 4 regarding, orthogonal collocation. Similar profiles were obtained for both the orthogonal collocation method and that implemented by the bvode function of Scilab.

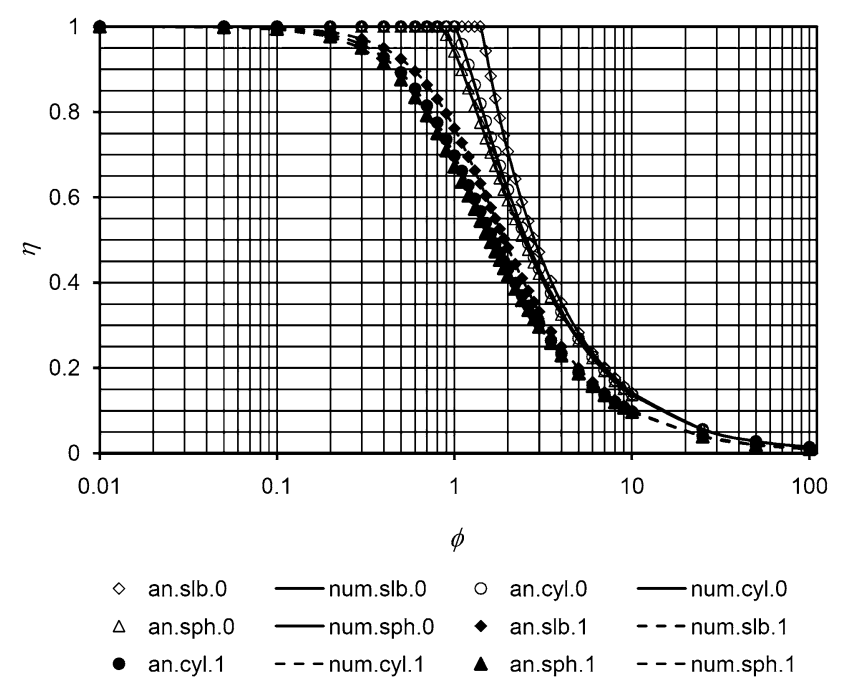

Fig. 5 Effectiveness factor versus Thiele modulus for: analytical (an.) and numerical (num.) methods; slab (slb.), cylinder ( $c y l$.$) , and$ sphere $(s p h$.) geometries; zero-order $(0)$ and first-order ( 1 ) kinetics
Results presented in Table 2 and Fig. 4 regarding orthogonal collocation suggest that another methodology must be employed to solve nonlinear BVP.

The results for orthogonal collocation methods on finite elements are presented in Table 3.

Table 3 shows an excellent accuracy for the calculated values of effectiveness and substrate concentration. The numerical values for the critical Thiele modulus also present good accuracy when analyzing zero-order kinetics. These calculations used 10 internal collocation points (orthogonal collocation method) within 10 equally spaced finite elements.

Although the analytical solution does not predict the occurrence of dead core for first-order kinetics, a critical value of Thiele modulus is determined by applying the orthogonal collocation on finite elements. This is due to the numerical error of the method itself. However, as shown in Fig. 4, for orthogonal collocation on finite elements, the calculated values of substrate concentration are approximately zero inside the predicted dead core and are within the numerical error.

Figure 5 shows the behavior of effectiveness factor as a function of the Thiele modulus for classical geometries (slb.-slab, cyl.-cylinder, sph.-sphere) and reaction kinetics of zero and first orders, calculated from the method of orthogonal collocation on finite elements.

According to the obtained results, the orthogonal collocation on finite elements was able to attain reliable values for both the internal effectiveness factor and substrate concentration profiles. This result indicates that the orthogonal collocation method on finite elements is able to solve similar BVPs to calculate the internal effectiveness factor for nonlinear kinetic expressions, such as the Michaelis-Menten kinetics and other derived expressions incorporating inhibition terms.

Table 4 Characteristics and capabilities of the numerical methods applied to solve the proposed BVPs

\begin{tabular}{|c|c|c|c|c|c|c|c|}
\hline & \multicolumn{4}{|c|}{ Shooting methods } & \multicolumn{3}{|c|}{ Collocation methods } \\
\hline & Adams & Stiff & RK & RK-4th & bvode function & Orthogonal & $\begin{array}{l}\text { Orthogonal on } \\
\text { finite elements }\end{array}$ \\
\hline Zero-order kinetics (for low $\phi$ ) & Solves & Solves & Solves & Solves & Solves & Solves & Solves \\
\hline Zero-order kinetics (for high $\phi$ ) & Solves & Solves & Solves & Solves & Solves & Solves & Solves \\
\hline First-order kinetics (for low $\phi$ ) & Solves & Solves & Solves & Solves & Solves & Solves & Solves \\
\hline First-order kinetics (for high $\phi$ ) & Diverges & Diverges & Diverges & Diverges & Inaccurate & Inaccurate & Solves \\
\hline Initial estimate & Without & Without & Without & Without & Unnecessary & Necessary & Necessary \\
\hline Convergence rate & High & Medium & High & High & Medium & Medium & Low \\
\hline Complexity of programming on Scilab & Low & Low & Low & Low & Medium & High & High \\
\hline
\end{tabular}




\section{Assessment of numerical methods}

Table 4 presents an assessment of characteristics and capabilities of the numerical methods used to solve the BVPs proposed in this study.

Table 4 shows that, among the various methods employed, the only one able to solve all of the proposed BVPs was the orthogonal collocation on finite elements method. However, for low values of the Thiele modulus, it may well be preferable to use the shooting method due to its programming simplicity and high convergence rate.

\section{Analysis of dead-core occurrence for the Michaelis- Menten kinetics}

Table 5 presents the results for orthogonal collocation methods on finite elements to solve the BVP concerning Michaelis-Menten kinetics.

According to Table 5, the orthogonal collocation on finite elements was able to determinate the parameters $a$, $\phi_{\text {crit }}$, and $\eta$ for all particle geometries (slab, cylinder, and sphere) and any values of $\beta$. The calculated values of both $a$ and $\phi_{\text {crit }}$ indicate that the dead-core occurrence for the
Table 5 Results for orthogonal collocation methods on finite elements to solve the BVP concerning Michaelis-Menten kinetics

\begin{tabular}{|c|c|c|c|c|c|c|c|c|c|}
\hline \multicolumn{5}{|c|}{$\beta=10^{-5}$} & \multicolumn{5}{|c|}{$\beta=10^{-3}$} \\
\hline$\alpha$ & $\phi$ & $\phi_{\text {crit }}$ & $a$ & $\eta$ & $\alpha$ & $\phi$ & $\phi_{\text {crit }}$ & $a$ & $\eta$ \\
\hline 1 & 0.5 & 1.4322 & 0 & 1.0000 & 1 & 0.5 & 2.7515 & 0 & 0.9999 \\
\hline 1 & 1 & 1.4322 & 0 & 1.0000 & 1 & 1 & 2.7515 & 0 & 0.9994 \\
\hline 1 & 2 & 1.4322 & 0.2826 & 0.7070 & 1 & 2 & 2.7515 & 0 & 0.7050 \\
\hline 1 & 4 & 1.4322 & 0.6413 & 0.3535 & 1 & 4 & 2.7515 & 0.3121 & 0.3525 \\
\hline 1 & 8 & 1.4322 & 0.8207 & 0.1768 & 1 & 8 & 2.7515 & 0.6561 & 0.1763 \\
\hline 2 & 0.5 & 1.0009 & 0 & 1.0000 & 2 & 0.5 & 1.4245 & 0 & 0.9998 \\
\hline 2 & 1 & 1.0009 & 0 & 0.9999 & 2 & 1 & 1.4245 & 0 & 0.9964 \\
\hline 2 & 2 & 1.0009 & 0.6133 & 0.6217 & 2 & 2 & 1.4245 & 0.3034 & 0.6159 \\
\hline 2 & 4 & 1.0009 & 0.8147 & 0.3320 & 2 & 4 & 1.4245 & 0.6547 & 0.3311 \\
\hline 2 & 8 & 1.0009 & 0.9089 & 0.1715 & 2 & 8 & 1.4245 & 0.8277 & 0.1710 \\
\hline 3 & 0.5 & 0.8167 & 0 & 1.0000 & 3 & 0.5 & 0.9663 & 0 & 0.9998 \\
\hline 3 & 1 & 0.8167 & 0.3843 & 0.9422 & 3 & 1 & 0.9663 & 0.0392 & 0.9400 \\
\hline 3 & 2 & 0.8167 & 0.7372 & 0.5933 & 3 & 2 & 0.9663 & 0.5358 & 0.5918 \\
\hline 3 & 4 & 0.8167 & 0.8755 & 0.3255 & 3 & 4 & 0.9663 & 0.7696 & 0.3245 \\
\hline 3 & 8 & 0.8167 & 0.9395 & 0.1703 & 3 & 8 & 0.9663 & 0.8851 & 0.1693 \\
\hline \multicolumn{5}{|c|}{$\beta=10^{\circ}$} & \multicolumn{5}{|c|}{$\beta=10^{2}$} \\
\hline$\alpha$ & $\phi$ & $\phi_{\text {crit }}$ & $a$ & $\eta$ & $\alpha$ & $\phi$ & $\phi_{\text {crit }}$ & $a$ & $\eta$ \\
\hline 1 & 0.5 & 61.2777 & 0 & 0.9584 & 1 & 0.5 & 86.5328 & 0 & 0.9249 \\
\hline 1 & 1 & 61.2777 & 0 & 0.8397 & 1 & 1 & 86.5328 & 0 & 0.7628 \\
\hline 1 & 2 & 61.2777 & 0 & 0.5427 & 1 & 2 & 86.5328 & 0 & 0.4829 \\
\hline 1 & 4 & 61.2777 & 0 & 0.2770 & 1 & 4 & 86.5328 & 0 & 0.2503 \\
\hline 1 & 8 & 61.2777 & 0 & 0.1385 & 1 & 8 & 86.5328 & 0 & 0.1252 \\
\hline 2 & 0.5 & 31.0911 & 0 & 0.9379 & 2 & 0.5 & 43.7509 & 0 & 0.8935 \\
\hline 2 & 1 & 31.0911 & 0 & 0.7743 & 2 & 1 & 43.7509 & 0 & 0.6989 \\
\hline 2 & 2 & 31.0911 & 0 & 0.4812 & 2 & 2 & 43.7509 & 0 & 0.4325 \\
\hline 2 & 4 & 31.0911 & 0 & 0.2593 & 2 & 4 & 43.7509 & 0 & 0.2342 \\
\hline 2 & 8 & 31.0911 & 0 & 0.1342 & 2 & 8 & 43.7509 & 0 & 0.1212 \\
\hline 3 & 0.5 & 20.9338 & 0 & 0.9259 & 3 & 0.5 & 29.4307 & 0 & 0.8771 \\
\hline 3 & 1 & 20.9338 & 0 & 0.7439 & 3 & 1 & 29.4307 & 0 & 0.6727 \\
\hline 3 & 2 & 20.9338 & 0 & 0.4637 & 3 & 2 & 29.4307 & 0 & 0.4174 \\
\hline 3 & 4 & 20.9338 & 0 & 0.2545 & 3 & 4 & 29.4307 & 0 & 0.2296 \\
\hline 3 & 8 & 20.9338 & 0 & 0.1328 & 3 & 8 & 29.4307 & 0 & 0.1200 \\
\hline
\end{tabular}


Michaellis-Menten kinetics is possible when verified certain conditions of diffusion-reaction within the catalytic particle.

It was also found that for values of $\beta$ close to zero, the parameters $a, \phi_{\text {crit }}$, and $\eta$ were close to those obtained for zero-order kinetics. For intermediate values of $\beta$, these parameters have values between those obtained for zeroand first-order kinetics, while for high values of $\beta$, the calculated parameters are close to those obtained for firstorder kinetics. These results were expected since the value of the constant $K$, which determines the behavior of Michaelis-Menten kinetics between zero- and first-order kinetics, is given by $K=\beta s_{0}$.

The simulated profiles of substrate concentration $(s)$ inside the particle for the Michaelis-Menten kinetics, $\alpha=3$ (spherical geometry) and $\phi=1$ are shown in Fig. 6.

Figure 6 shows that the simulated profiles of $s$ are between the profiles for first- and zero-order kinetics. These result agree with the asymptotic analysis of Eq. 10 presented in the mathematical modeling section. For
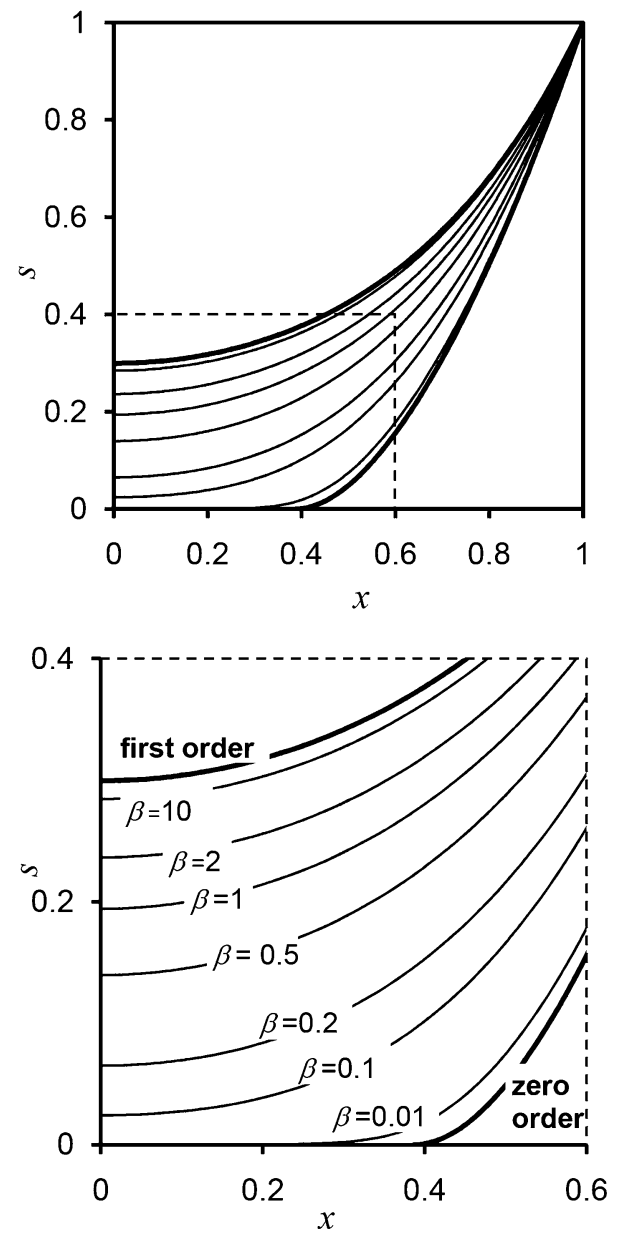

Fig. 6 Simulated profiles of substrate concentration $(s)$ inside the particle for the Michaelis-Menten kinetics, $\alpha=3$ (spherical geometry) and $\phi=1$ diffusion-reaction conditions simulated in Fig. 6, it can be observed that the dead core occurs for low values of $\beta$ (e.g., $\beta \leq 0.01)$.

\section{Effectiveness factor for the Michaelis-Menten kinetics}

Using the orthogonal collocation method on finite elements, it was possible to calculate the effectiveness factor for three geometries and for any values of the Thiele modulus and dimensionless Michaelis-Menten constant $(\beta)$, as shown in Fig. 7.

Figure 7 shows that, for high values of $\beta$, the calculated values of the effectiveness factor are close to those calculated for first-order kinetics, whereas for low $\beta$ values, they are close to those calculated for zero-order kinetics. This result was expected, given that the Michaelis-Menten kinetics incorporates the behavior of those kinetic equations. These results confirm the validity of the orthogonal collocation method on finite elements to solve the boundary value problem associated with Michaelis-Menten
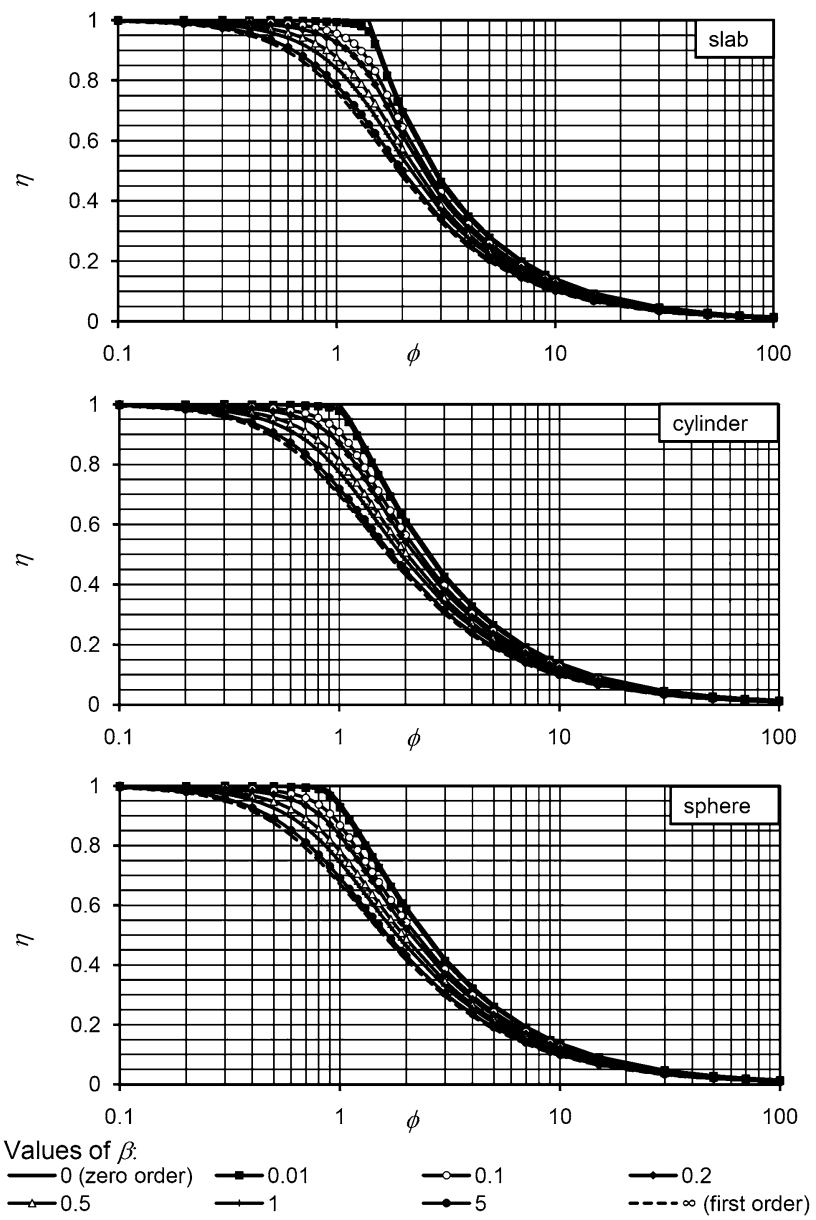

Fig. 7 Effectiveness factor versus Thiele modulus for the MichaelisMenten kinetics 
kinetics, since that the numerical solutions were identical to the analytical solutions for the limit cases.

\section{Conclusions}

According to the present study's results, the following conclusions can be drawn:

- For low values of the Thiele modulus, the use of shooting methods is preferred due to its programming simplicity and high convergence rate;

- For high values of the Thiele modulus, the shooting methods can be divergent;

- For high values of the Thiele modulus, the orthogonal collocation method and that implemented by the bvode function of Scilab can result in inaccurate values of the effectiveness factor;

- Among the methods studied in this article, the orthogonal collocation method on finite elements was the most effective and promising to solve nonlinear boundary value problems associated with the modeling of diffusion-reaction processes in catalytic particles containing immobilized enzymes;

- The orthogonal collocation method on finite elements was successfully applied to the case of the MichaelisMenten kinetics, suggesting that this method can be extended to other types of kinetic expressions, such as those that incorporate inhibition effects;

- For the Michaelis-Menten kinetics, the dead core can occur when verified certain conditions of diffusionreaction within the catalytic particle;

- The application of the concepts and methods presented in this study will allow for a more generalized analysis and more accurate designs of heterogeneous enzymatic reactors.

\section{References}

1. Šekuljica NŽ, Prlainović NŽ, Jovanović JR, Stefanović AB, Djokić VR, Dušan ŽM, Knežević-Jugović ZD (2016) Immobilization of horseradish peroxidase onto kaolin. Bioproc Biosyst Eng. doi:10.1007/s00449-015-1529-x

2. Edet E, Ntekpe M, Omereji S (2013) Current trend in enzyme immobilization: a review. Int J Mod Biochem 2:31-49

3. Illanes A (2008) Enzyme biocatalysis: principles and applications. Springer, Netherlands

4. Bailey JE, Ollis DF (1986) Biochemical engineering fundamentals. McGraw-Hill, New York

5. Doran PM (2013) Bioprocess engineering principles. Academic Press, Waltham

6. Fogler HS (2005) Elements of chemical reaction engineering. Prentice Hall PTR, Upper Saddle River
7. Oliveira SC (1999) Evaluation of effectiveness factor for immobilized enzymes using Runge-Kutta-Gill method: how to solve mathematical undetermination at particle center point? Bioproc Eng. doi:10.1007/s004490050579

8. Granato MA, Queiroz LC (2003) Dead core in porous catalysts: modeling and simulation of a case problem using Mathematica. In: Proceedings of the workshop modelling and simulation in chemical engineering, CIM-Centro Internacional de Matemática, Coimbra, 30 June-4 July 2003

9. Bird RB, Stewart WE, Lightfoot EN (2002) Transport phenomena. Wiley, New York

10. Froment GF, Bischoff KB, De Wilde J (2011) Chemical reactor analysis and design. Wiley, Hoboken

11. Mahalakshmi M, Hariharan G (2016) An efficient Chebyshev wavelet based analytical algorithm to steady state reaction-diffusion models arising in mathematical chemistry. J Math Chem. doi:10.1007/s10910-015-0560-0

12. Devi MR, Sevukaperumal S, Rajendran L (2015) Non-linear reaction diffusion equation with Michaelis-Menten kinetics and Adomian decomposition method. Appl Math. doi:10.5923/j.am. 20150501.04

13. Shanthi D, Ananthaswamy V, Rajendran L (2013) Analysis of non-linear reaction-diffusion processes with Michaelis-Menten kinetics by a new Homotopy perturbation method. Nat Sci. doi:10.4236/ns.2013.59128

14. Joy RA, Meena A, Loghambal S, Rajendran L (2011) A twoparameter mathematical model for immobilized enzymes and Homotopy analysis method. Nat Sci. doi:10.4236/ns.2011.37078

15. Hindmarsh AC (1983) In: Stepleman RS et al (eds) Scientific computing. IMACS Transactions on Scientific Computation, Amsterdam

16. Brown PN, Hindmarsh AC (1989) Reduced storage matrix methods in stiff ODE systems. Appl Math Comput. doi:10.1016/ 0096-3003(89)90110-0

17. Description and use of LSODE, the Livermore solver for ordinary differential equations (1993) NASA, Livermore. https://computa tion.llnl.gov/casc/nsde/pubs/u113855.pdf. Accessed 16 Feb 2016

18. Shampine LF (1996) Some practical Runge-Kutta formulas. Math Comput. doi:10.2307/2008219

19. Shampine LF, Watts HA (1980) DEPAC-design of a user oriented package of ODE solvers. Sandia National Laboratories, Albuquerque

20. Fehlberg E (1970) Klassische Runge-Kutta-Formeln vierter und niedrigerer Ordnung mit Schrittweiten-Kontrolle und ihre Anwendung auf Wärmeleitungs probleme. Computing. doi:10. 1007/BF02241732

21. Ascher U, Christiansen J, Russell RD (1981) Collocation software for boundary-value ODEs. ACM T Math Softw. doi:10. $1145 / 355945.355950$

22. Bader G, Ascher U (1987) A new basis implementation for a mixed order boundary value ode solver. SIAM J Sci Stat Comp. doi: $10.1137 / 0908047$

23. Ascher U, Christiansen J, Russell RD (1979) A collocation solver for mixed order systems of boundary value problems. Math Comput. doi:10.1090/S0025-5718-1979-0521281-7

24. Deboor C, Weiss R (1980) SOLVEBLOK: a package for solving almost block diagonal linear systems. ACM T Math Softw. doi: $10.1145 / 355873.355880$

25. Villadsen J, Michelsen ML (1978) Solution of differential equation models by polynomial approximation. Prentice-Hall, Englewood Cliffs

26. Wylie CR, Barret LC (1982) In: Wylie CR, Barret LC (eds) Advanced engineering mathematics. McGraw-Hill, Singapore 